\title{
Comparison of two FDA-approved interspinous spacers for treatment of lumbar spinal stenosis: Superion versus X-STOP - a meta-analysis from five randomized controlled trial studies
}

He Zhao ${ }^{1+}$, Li-Jun Duan ${ }^{2+}$, Yu-Shan Gao ${ }^{3}$, Yong-Dong Yang ${ }^{1}$, Ding-Yan Zhao ${ }^{1}$, Xiang-Sheng Tang ${ }^{4}$, Zhen-guo Hu', Chuan-Hong Li ${ }^{1}$, Si-Xue Chen ${ }^{1}$, Tao Liu ${ }^{1}$ and Xing Yu${ }^{1 *}$

\section{Abstract}

Background: Decompressive laminectomy (DI) is a standard operation for lumbar spinal stenosis (LSS) patient with severe claudication symptoms for many years. However, patients whose symptom severity does not meet undergoing invasive surgery make therapeutic options into dilemma. Interspinous spacers (ISP) bridge the gap between surgical interventions and CC in management of LSS. In our study, we aim to systematically assess the two FDA-approved interspinous spacers for treatment of lumbar spinal stenosis: Superion versus X-STOP.

Methods: Electronic databases, including PubMed, Embase, MEDLINE, Cochrane Library were searched to retrieve clinical trials concerning the comparison between Superion and X-STOP in treatment for lumbar spinal stenosis before April 2017. The following outcome measures were extracted: (1) Zurich Claudication Questionnaire (ZCQ) patient satisfaction score, (2) axial pain severity, (3) extremity pain severity, (4) back-specific functional impairment, (5) reoperation, and (6) complication. The data anakysis was conducted with Review Manager 5.3.

Results: Five randomized controlled trials (RCTs) with 1118 patients were included in this meta-analysis. The pooled analysis indicated that the Superion group is superior to X-STOP in axial pain severity (SMD: 0.03; 95\% CI 0.15, 0.45; $\left.p<0.0001, P^{2}=41 \%, p=0.16\right)$, ZCQ patient satisfaction score (SMD: $0.23 ; 95 \% \mathrm{Cl} 0.08,0.38 ; p=0.002, P=0 \%, p=0.61$ ). However, Superion group showed similarity outcome in extremity pain severity (SMD: $0.18 ; 95 \% \mathrm{Cl}-0.06,0.43 ; p=0.14$, $P^{2}=62 \%, p=0.05$ ), back-specific functional impairment (SMD: $0.04 ; 95 \% \mathrm{Cl}-0.10,0.19 ; p=0.56, P^{2}=0 \%, p=0.77$ ), reoperation rate (RR: 1.10; $95 \% \mathrm{Cl} 0.82,1.48 ; p=0.51, P^{2}=19 \%, p=0.30$ ), and complication (RR: $0.98 ; 95 \% \mathrm{Cl} 0.63,1.53$; $p=0.92, P^{2}=0 \%, p=0.83$ )

Conclusion: Both the Superion and X-STOP interspinous spacers can relieve symptoms of LSS. In addition, the Superion spacer max represent a promising spacer for patient with LSS. As we know, the effectiveness and safety of ISP is still considered investigational and unfavor clinical results in the medical literature may continue to limit the appeal of IPS to many surgeons in the future. However, because of the advantage of IPS technique, it will win a wide place in the future degenerative lumbar microsurgery.

Keywords: Superion, X-STOP, Follow-up, Meta-analysis

\footnotetext{
* Correspondence: yuxingbucm@sina.com

${ }^{\dagger}$ Equal contributors

'Department of Orthopedics III, Dongzhimen Hospital Affiliated to Beijing

University of Chinese Medicine, No. 5 Haiyuncang Street, Dongcheng District,

Beijing 100700, China

Full list of author information is available at the end of the article
} 


\section{Background}

Lumbar spinal stenosis (LSS) is characterized as narrowing of the spinal canal, lateral recesses, or neuroforamina, resulting in entrapment of the cauda equine and exiting nerve roots due to hypertrophy of the osseous and soft tissue structures surrounding the lumbar spinal canal [1]. The classic manifestation is intermittent neurogenic symptom including low back and leg pain exacerbated by standing, ambulatory, or trunk extension. With the progression of compression, neurogenic claudication secondary to LSS may occur. The annual incidence of LSS is 5 per 100,000 people, most commonly affects the middle-aged and elderly population $[2,3]$. As society grows older, LSS becomes one of the main causes of medical visits and responsible for a tremendous impact in developed countries, from a social, economic, and medical point of view. Many studies indicate that conservative care (CC) for treatment of LSS cannot hinder the progression of the disease. Thus, decompressive laminectomy (DI) is a standard operation for LSS patient with severe claudication symptoms who fail to CC. However, patients whose symptom severity does not meet undergoing invasive surgery make therapeutic options into dilemma [4-6]. Interspinous spacers (ISP) bridge the gap between surgical interventions and CC in the management of LSS representing a promising minimally invasive procedure. Those devices are implanted between contiguous spinous processes via minimal surgical incision and alleviate neurogenic claudication symptoms by limiting spinal extension. Nowadays, various interspinous spacers such as Coflex, Wallis, DIAM, X-STOP, and Extersure Felix trial are still practiced in clinical [7]. It is noteworthy that, in 2005, the X-STOP became the first Food and Drug Administration (FDA)-approved interspinous process spacer for treatment of neurogenic claudication secondary to LSS. Since then, no other interspinous process spacers have received FDA approval. However, 10 years later, Superionas the second "stand-alone" ISP approved by the FDA on May 20, 2015, got the relay baton after X-STOP was ceased to sale and distribution by its manufacturer (Medtronic, Inc., Minneapolis, MN, USA) in 2015. Although two stand-alone IPSs possessed similar mechanism in treatment for lumbar spinal stenosis, there were inherent differences in surgical placement technique and device design between two spacers. Different from X-STOP, the distinct merits of Superion procedure is minimally/invasive approach with smaller blood loss and shorter hospital stay [8], and it does not cause substantial alterations or disruptions to the spinal anatomy, which likely reduces the complexity of future surgical options in the event that revision becomes necessary to

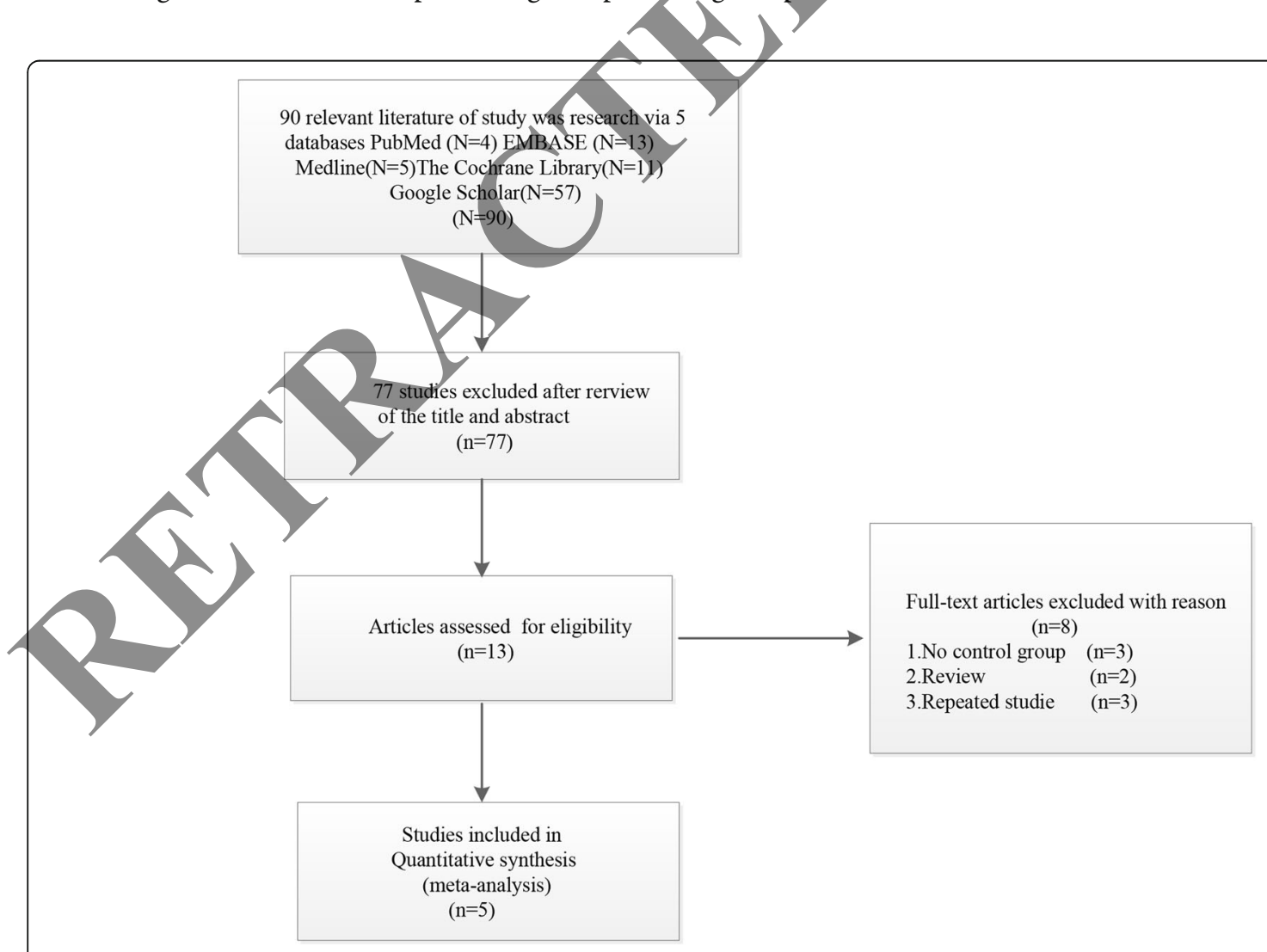

Fig. 1 Flow chart of study selection process 
Table 1 Characteristics of included studies

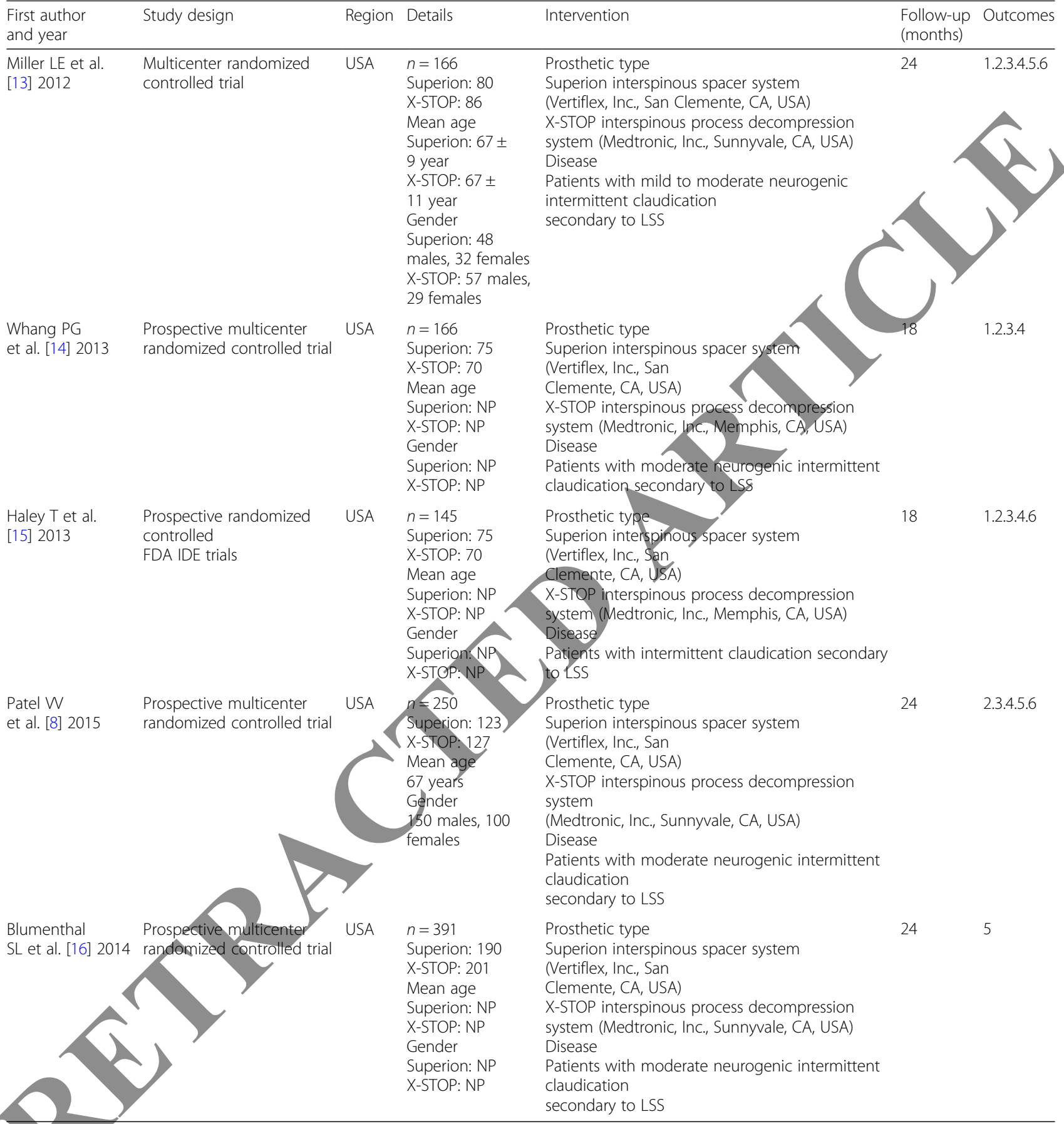

Note: ZCQ patient satisfaction score, 2 axial pain severity, 3 extremity pain severity, 4 back-specific functional impairment, 5 reoperation, 6 complication

address progressive degenerative changes and/or reemergence of symptoms. Besides the operative benefits, there are also biomechanical properties compared with X-STOP on the basis of the radiographical data demonstrating Superion group generated significant lower rate of dislodgements and migrations than in the X-STOP group. In terms of device design, the wings of the $\mathrm{X}-\mathrm{STOP}$ is more slender than Superion result in providing less stability between the spinous processes and exhibit greater pain, loss of function, and a higher rate of second operation [9]. Although, many previous published articles are mainly focused on comparing traditional decompressive surgery with interspinous spacer, we are aiming to compare those two types of interspinous spacer in treatment of lumbar spinal stenosis [10-12]. The purpose of this meta-analysis is to compare 


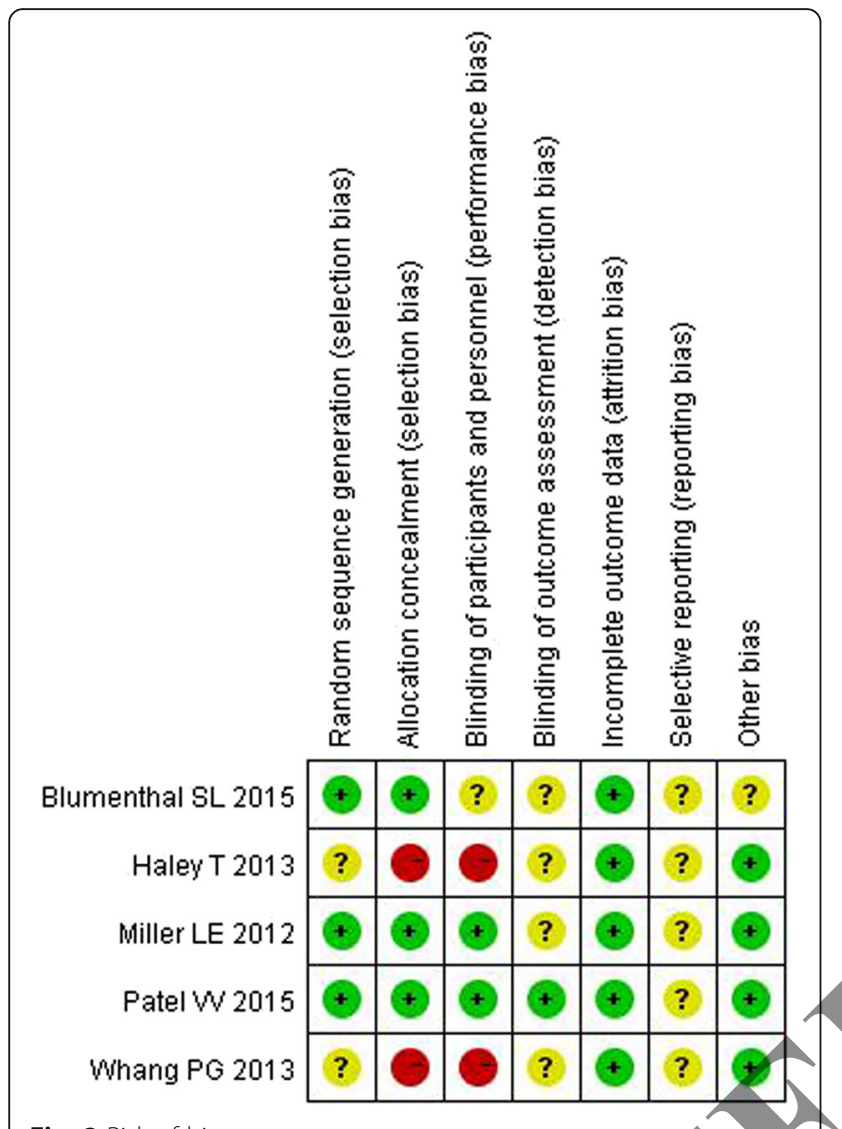

Fig. 2 Risk of bias summary

the clinical outcomes in patients treated with two FDA-approved interspinous spacers.

\section{Methods}

\section{Search strategy}

To search all of the relevant literature, we systematically searched for literature published in the database (PubMed, Embase, MEDLINE, Cochrane Library, Google Scholar). The search terms were subjected to the following: "Superion," "X-STOP," "interspinous spacer," and "lumbar spinal stenosis" with various combinations of the operators "AND," "NOT," and "OR." There was a restriction of study design which was the randomized controlled trial ( $R C T$ ) published between January 2000 and April 2017. The restricted language was English. The references cited in the relevant articles were also reviewed.

\section{Inclusion criteria}

All studies on treatment of lumbar spinal stenosis were reviewed. The criteria for inclusion of an article were (1) Superion compared with X-STOP for treatment of lumbar spinal stenosis, (2) a randomized controlled trial (RCT), and (3) studies with follow-up more than 6 months. Two authors assessed the potentially eligible studies independently; any disagreement was discussed and resolved by a third independent authør.

\section{Data extraction}

The following data were extracted by two authors independently using a purpose-designed form: First author and year, study, design, region, details, intervention follow-up (months), and outcomes:

1) Zurich Claudication Questionnaire (ZCQ) patient satisfaction score

2) Axial pain severity

3) Extremity pain seyerity

4) Back-specific functional impairment

5) Reoperation

6) Complication

Disagreement between the two reviewers was settled by the third reviewer. If any disagreements existed, a third author was consulted for a discussion until consensus was reached.

\section{Quality assessment}

The quality of the studies was independently assessed by the two authors according to a 12-item scale recommended by the Cochrane Back Review Group. If at least six of the 12 criteria, including randomization, allocation concealment, blinding (of the patients, assessors, and surgeons), similar baseline, selective reporting, loss to follow-up, patient compliance, similar timing, and intention-to-treat (ITT) analysis, were met without serious flaws, the studies were rated as having "low risk of bias." Otherwise, the studies were rated as having "high risk of bias."

\section{Statistical analysis}

The risk ratio (RR) and the corresponding 95\% confidence interval (CI) were assessed for the dichotomous

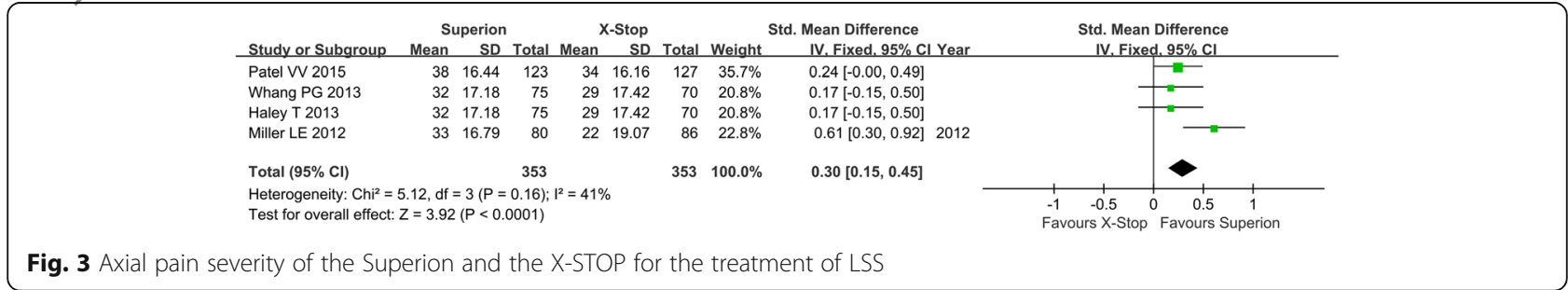




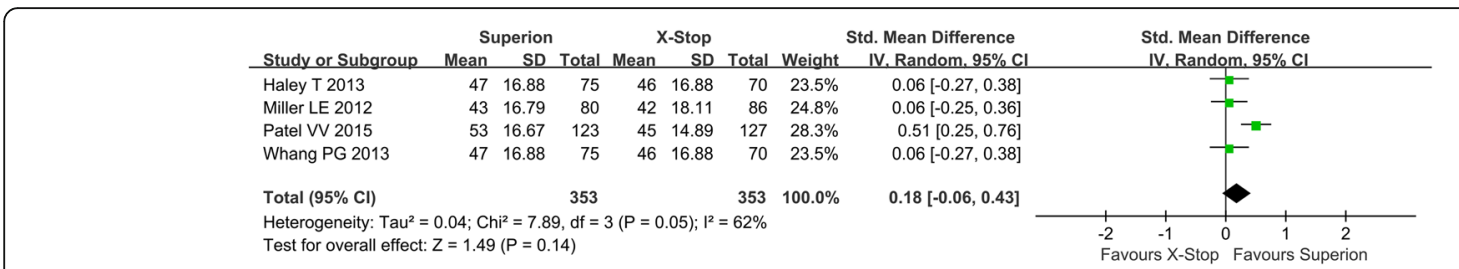

Fig. 4 Extremity pain severity of the Superion and the X-STOP for the treatment of LSS

outcomes, and the standardized mean difference (SMD) and 95\% CI were assessed for the continuous outcomes. The chi-square test and Higgin's $I^{2}$ test were used to evaluate the heterogeneity. A $p$ value less than 0.05 for the chi-square test or $I^{2}$ values exceeding $50 \%$ indicated substantial heterogeneity. A fixed-effect model was used if significantly statistical heterogeneity was absent; otherwise, a random-effect model was applied. Because of the limited number of included studies, we did not assess the possibility of publishing bias. We used Review Manager Software (RevMan Version 5.3, The Cochrane Collaboration) to conduct the statistical analysis.

\section{Results}

Flow chart for the inclusion of studies is shown in Fig. 1. The literature search initially yielded 36 relevant trials from PubMed $(N=4)$, Embase $(N=13)$, MEDLINE $(N=5)$, The Cochrane Library $(N=11)$, and Google Scholar $(N=57)$. After we reviewed the titles and abstracts of all 90 trials, 77 trials were excluded. We continued to refine the 13 studies under provident review and excluded 8 articles because of the following reason: (1) no control group $(n=3)$, (2) review $(n=2)$, and (3) repeated studies $(n=3)$.) Finally, 5 RCTs with 1118 patients were included (Fig. 1). We recorded the characteristics of the 5 included trials, as well as the details of the clinical outcome measurement (Table 1).

\section{Methodological study quality assessment}

A summary of methodological domain assessment for each study is detailed in Fig 2 .

\section{Axial pain severity}

Four studies reported axial pain severity of lumbar spinal stenosis after the operation. Because no heterogeneity existed among studies, the fixed model was used (Fig. 2). In forest plots, the overall effect indicated significant differences between the Superion and the X-STOP group (SMD: $0.03 ; 95 \%$ CI $0.15,0.45 ; p<0.0001, I^{2}=41 \%$, $p=0.16)$ (Fig. 3).

\section{Extremity pain severity}

Four studies reported extremity pain severity postoperatively, and the random model was used due to heterogeneity in studies. The pooled results showed that the Superion group had no better improvement than X-STOP (SMD: 0.18; 95\% CI $-0.06,0.43 ; p=0.14$, $I^{2}=62 \%, p=0.05$ ) (Fig. 4).

\section{Back-specific functional impairment}

Four studies reported back-specific functional impairment after the operation. No heterogeneity existed among studies, and the fixed model was used in metaanalysis. In the forest plots, the overall effect indicated no significant differences between the Superion and the X-STOP group (SMD: 0.04; 95\% CI - 0.10, 0.19; $p=0.56$, $I^{2}=0 \%, p=0.77$ ) (Fig. 5).

\section{Reoperation rate}

The reoperation rate was reported in four studies. No heterogeneity existed among studies, and the fixed model was used in the meta-analysis. In the forest plots, the overall effect indicated no significant differences between the Superion and the X-STOP group (RR: 1.10; 95\% CI 0.82, 1.48; $p=0.51, I^{2}=19 \%, p=0.30$ ) (Fig. 6).

\section{ZCQ patient satisfaction score}

Four studies reported the ZCQ patient satisfaction score postoperatively. Due to no heterogeneity among studies, the fixed model was used. The overall effect showed that there were significant differences between the Superion and the X-STOP group. (SMD: 0.23; 95\% CI 0.08, 0.38; $p=0.002, I^{2}=0 \%, p=0.61$ ) (Fig. 7).

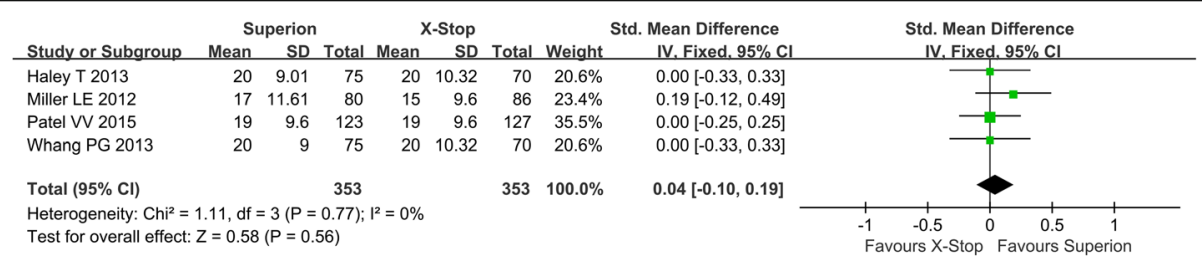

Fig. 5 Back-specific functional impairment of the Superion and the X-STOP for the treatment of LSS 


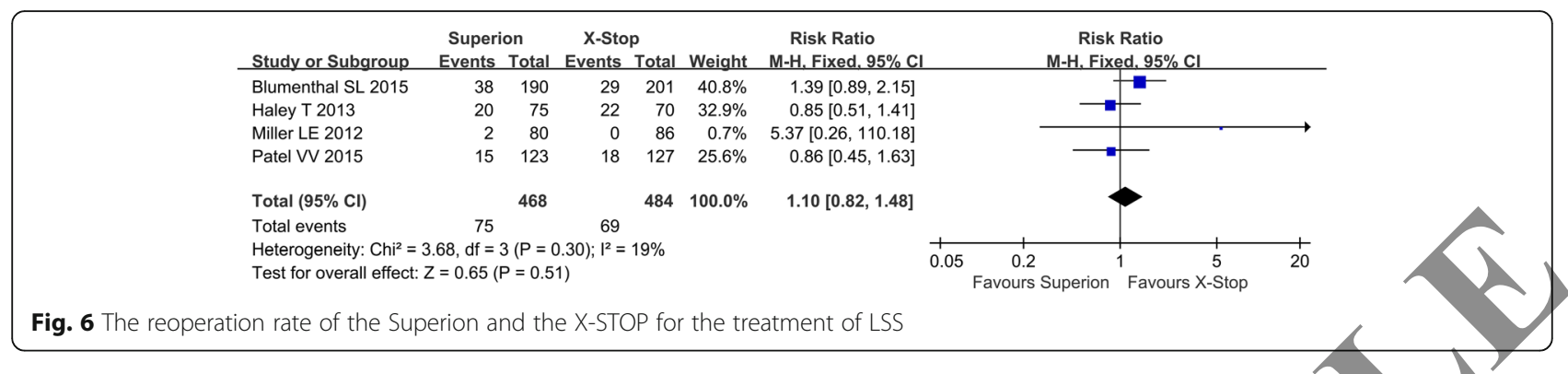

\section{Complication}

The complication was reported in two studies. No heterogeneity existed between two studies, and the fixed model was used in the meta-analysis. In the forest plots, the overall effect indicated no significant differences between the Superion and the X-STOP group (RR: 0.98; 95\% CI 0.63, 1.53; $p=0.92, I^{2}=0 \%, p=0.83$ ) (Fig. 8).

\section{Discussion}

The results of this meta-analysis demonstrated that the Superion spacer provides the similar clinical outcome in extremity pain severity, back-specific functional impairment, reoperation rate, and complication back function. However, Superion showed a better outcome than XSTOP in axial pain severity and ZCQ patient satisfaction score. The similarity mechanism in two types of ISP may account for the pooled outcome. The mechanism of various ISPs in current spine field includes five points [17-19]: (1) enlargement of the spinal canal area, (2) increase of the neural foramina area, (3) unloading of the posterior annulus and intradiscal pressure, (4) distraction of interspinous distance, and (5) strength of the spinous processes.

Since the 1950s, the first ISP was designed and applied by Konwles [20] in clinical with many defects. For three decades of progress, the first modern ISP, Wallis spacer, was developed by Senegas [21] in the early 1980s. Moreover, as a new procedure, the ISP becomes an alternative option for the patient and makes a profound influence in treating LSS. Now, designs of ISP vary from standalone spacers represented by the X-STOP to "dynamic" spacers represented by the Coflex, respectively. The X-STOP was approved by the US Food and Drug Administration based on laboratory, mechanical, cadaver, and clinical studies in 2005 for treatment of patients aged above 50 suffering from neurogenic intermittent claudication secondary to a confirmed diagnosis of LSS. Additionally, many studies have shown favorable outcomes compared to decompression surgery (with or without fusion) [22, 23]. According to a recent report, X-STOP was ceased to sale and distribution by its manufacturer (Medtronic, Inc. Minneapolis, MN, USA) in 2015, leaving the Superion as the de facto clinical option for surgeons and their patients. From experiment to clinical practice paradigm for remedy of LSS, the Superion as the second generation of stand-alone ISP was approved by the US Food and Drug Administration for commercial distribution in the USA on May 20, 2015. Both Superion and X-STOP spacers, albeit similarities in the mechanism of action and clinical outcomes, have distinct differences in device design and surgical technique. Superion spacer (size range from 8 to $16 \mathrm{~mm}$ ) is inserted through a cannula placed between adjacent spinous processes without dissection of the spinal musculature. Compared to the X-STOP spacer with large surgical exposure, the minimally invasive nature of the Superion spacer contributes to smaller blood loss, less operative time, and shorter hospitalization. In addition to its small incision, the Superion procedure avoids generating large scar tissue around symptomatic levels which may reduce the intricacy of future revision, removal, or further decompression surgery [24]. The data from previous 2-year follow-up studies suggested that both DI and the Superion procedure provide effective and durable symptom relief of claudication symptom. For back-pain severity, the Superion group and DI group obtain an average percentage improvement of 65 and $52 \%$. For the leg pain severity, the average percentage change with the spacer is $70 \%$, and laminectomy is $62 \%$ in comparison to [8]. Although patients with IPS

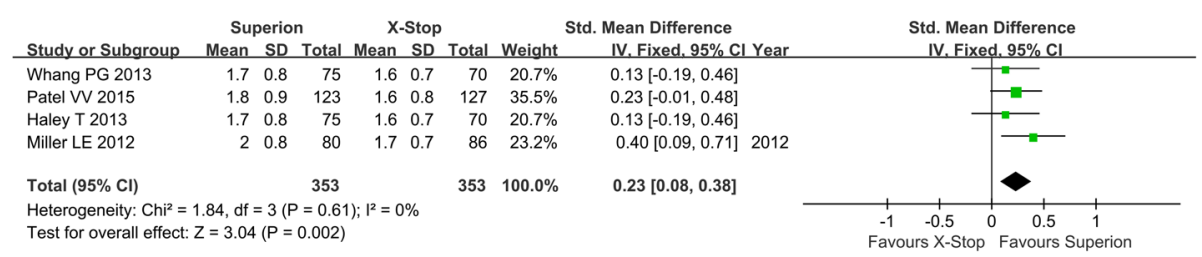

Fig. 7 ZCQ patient satisfaction score of the Superion and the X-STOP for the treatment of LSS 


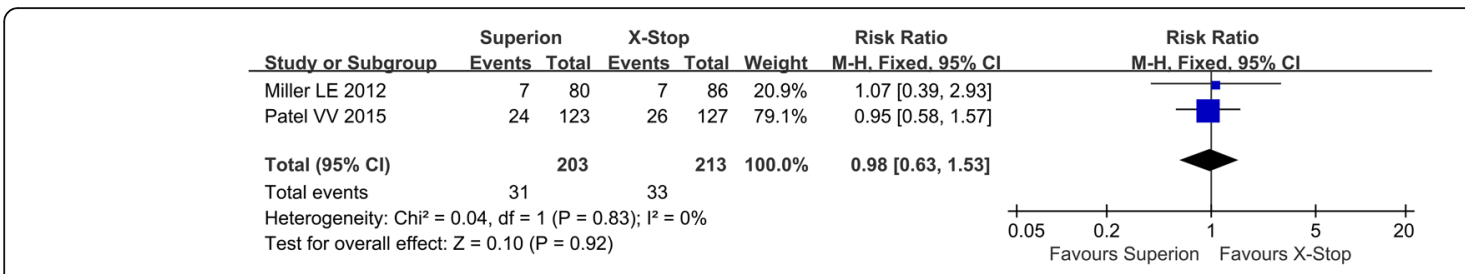

Fig. 8 Complication of the Superion and the X-STOP for the treatment of LSS

effectively ameliorate mild to moderate symptom of LSS in midterm and with the relatively small trauma of surgery, the cost-effectiveness issue for IPS should be considered. Parker et al. [25] use Markov model to evaluate three strategies of care for LSS. The study shows that CC has the lowest cost at $\$ 10,540$ and the lowest quality-adjusted life year increase, while ISP and DI were nearly identical at about $\$ 13,950$ and also at qualityadjusted life years. They indicated that surgical care, especially in ISP procedure, provides superior value versus sustained CC in treating LSS. However, another study reveals that for the 1-level procedure, a mean cost of $\$ 9291$ for DI, $\$ 7900$ for ISP, and \$3478 for CC, and for 2-level $\$ 13,429$ was a cost for ISP, and the other two treatments remain unchanged [26]. Despite the merits of the ISP were mentioned above, it is noteworthy that not only complications associated with process fracture, dislodgements, and migrations of the spacer, heterotopic ossification [27], brought barriers to its safety but also the exceeding indication restricted its clinical application.

It is noteworthy that both IPD and decompressiye surgery are current treatment strategies for lumbar spinal stenosis. However, which procedure can bring patients better outcomes is still pending. Moojen et al [28, 29] showed that, at 2 years, the success rate according to the ZCQ for the IPD group [69\% (95\% CI 57-78\%)] did not show a significant difference compared with decompression [60\% (95\% C1 48-71\%) $p=0.2]$. Furthermore, longterm VAS back pain was significantly higher $[36 \mathrm{~mm}$ on a $100-\mathrm{mm}$ scale ( $95 \%$ CI $24-48$ )] in the IPD group compared to the decompression group [28 mm (95\% CI 2334) $p=0.04]$. But the IPD group caused higher reoperation rate $(29 \%)$ than that in the decompression group (8\%) in the early post-surgical period. In 2013, Strömqvist et al. [22] conducted a trial to compare the X-STOP with conventional decompression in patients with neurogenic intermittent claudication. The results showed that the clinical improvement of two procedures were similar at 6,12 , and 24 months follow-up. Similarly, patients suffered from a higher rate of reoperations in the X-STOP group (26\%) than decompression surgery $(6 \%)$ at endpoint. Intriguingly, in two meta-analysis reviews $[30,31]$, we found that there is no significant difference between the IPD and decompression surgery for effective indicator such as Visual Analog Scale, Oswestry Disability Index, and Roland Disability Questionnaire and Complications. In addition, for the reoperation rates, both studies draw the same conclusion that the decompression group is lower than the IPD group.

This meta-analysis has a few limitations. According to our research results and inclusion criteria, five RCT studies are incladed. A few RCTs and incomplete data may reduce the quality of evidence and strength of analysis. Blinding of patients and surgeons are difficult to evaluate surgical effect in a clinical trial. Inadequate blinding is reported to generate $15 \%$ overestimation of treatment effect.

\section{Conclusions}

Based on the above study from 18 to 24 months of clinical pooled outcome, we concluded that both the Superion and X-STOP interspinous spacers can relieve symptoms of LSS. In addition, the pooled results indicate that Superion shows advantage than X-STOP in axial pain severity and ZCQ patient satisfaction score, whereas similar in other outcomes. The Superion spacer may represent a promising spacer for patient with LSS owing to its nature of minimally invasive, easily operable, and less damage. Because of the advantage of IPS technique, it would win a wide place in the future degenerative lumbar microsurgery.

\section{Abbreviations}

CC: Conservative care; Cl: Confidence interval; DI: Decompressive laminectomy; ISP: Interspinous spacers; LOE: Level of evidence; LSS: Lumbar spinal stenosis; RCT: Randomized controlled trial; RR: Risk ratio; SMD: Standardized mean difference

\section{Acknowledgements}

None.

\section{Funding}

None.

Availability of data and materials

All data and materials were presented in the main paper.

\section{Authors' contributions}

$\mathrm{XY}$ contributed to the design of the study. $\mathrm{HZ}$ and LJD were responsible for the data collection, data analysis, and drafting of the manuscript. YSG, YDY, and DYZ contributed to analyzing the data with regard to its potential clinical significance. XST and SXC conceived the meta-analysis and participated in its design and coordination. $\mathrm{ZH}, \mathrm{CHL}$, and TL screened the titles and abstracts of 
eligible citations and determined if they met the inclusion criteria to this meta-analysis. All authors read and approved the final manuscript.

\section{Ethics approval and consent to participate}

Not applicable

\section{Consent for publication}

Not applicable

\section{Competing interests}

The authors declare that they have no competing interests.

\section{Publisher's Note}

Springer Nature remains neutral with regard to jurisdictional claims in published maps and institutional affiliations.

\section{Author details}

'Department of Orthopedics III, Dongzhimen Hospital Affiliated to Beijing University of Chinese Medicine, No. 5 Haiyuncang Street, Dongcheng District, Beijing 100700, China. ${ }^{2}$ Department of Orthopedics, Bayannaoer City Hospital, No. 98 Wulanbuhe Street, Lin He District, Bayannaoer 015000, China. ${ }^{3}$ School of Basic Medical Sciences, Beijing University of Chinese Medicine, No. 11 East Road North 3rd Ring, Chao Yang District, Beijing 100029, China. ${ }^{4}$ Department of Orthopedics, China-Japan Friendship Hospital Affiliated to Beijing University of Chinese Medicine, No. 11 East Road North 3rd Ring, Chao Yang District, Beijing 100029, China.

\section{Received: 30 November 2017 Accepted: 8 February 2018} Published online: 02 March 2018

\section{References}

1. Katz JN, Harris MB. Lumbar spinal stenosis. N Engl J Med. 2008;358(8):818-25.

2. Global Burden of Disease Study 2013 Collaborators. Global, regional, and national incidence, prevalence, and years lived with disability for 301 acute and chronic diseases and injuries in 188 countries, 1990-2013 analysis for the Global Burden of Disease Study 2013. Lancet. 2015: 386(9995):743-800.

3. Markman JD, Gaud KG. Lumbar spinal stenosis in older adults: current understanding and future directions. Clin Geriatr Med. 2008;24(2):369-88.

4. Jakola AS, Sørlie A, Gulati S, Nygaard OP, Lydersen S, Solberg T. Clinical outcomes and safety assessment in elderly patients undergoing decompressive laminectomy for lumbar spinal stenosis: a prospective study. BMC Surg. 2010;10:34.

5. Weinstein JN, Tosteson TD, Lurie JD, Tosteson AN, Blood E, Hanscom B, Herkowitz $\mathrm{H}$, et al. Surgical versus nonsurgical therapy for lumbar spinal stenosis. N Engl J Med. 2008;358(8):794-810

6. Deyo RA, Mirza SK, Martin BI, Kreuter W, Goodman DC, Jarvik JG. Trends, major medical complications, and charges associated with surgery for lumbar spinal stenosis in older adults. JAMA. 2010;303(13):1259-65.

7. Gala RJ, Russo GS, Whang PG. Interspinous implants to treat spinal stenosis. Curr Rev Musculoskelet Med. 2017;10(2):182-8

8. Patel W, Whang PG, Haley TR, Bradley WD, Nunley PD, Davis RP, et al. Superion interspinous process spacer for intermittent neurogenic claudication secondary to moderate lumbar spinal stenosis: two-year results from a randomized controlled FDA-IDE pivotal trial. Spine. 2015:40(5):275-82

Loguidice V,Bini W, Shabat S, Miller LE, Block JE. Rationale, design and linical performance of the Superion ${ }^{\oplus}$ interspinous spacer: a minimally invasive implant for treatment of lumbar spinal stenosis. Expert Rev Med Devices. 2011:8(4):419-26.

10. Deyo RA, Martin Bl, Ching A, Tosteson AN, Jarvik JG, Kreuter W, et al. Interspinous spacers compared to decompression or fusion for lumbar stenosis: complications and repeat operations in the Medicare population. Spine (Phila Pa 1976). 2013;38(10):865-72.

11. Kabir SM, Gupta SR, Casey AT. Lumbar interspinous spacers: a systematic review of clinical and biomechanical evidence. Spine (Phila Pa 1976). 2010; 35(25):E1499-506

12. Beyer F, Yagdiran A, Neu P, Kaulhausen T, Eysel P, Sobottke R. Percutaneous interspinous spacer versus open decompression: a 2-year follow-up of clinical outcome and quality of life. Eur Spine J. 2013;22(9):2015-21.
13. Miller LE, Block JE. Interspinous spacer implant in patients with lumbar spinal stenosis: preliminary results of a multicenter, randomized, controlled trial. Pain Res Treat. 2012;2012:8235091.

14. Whang PG, Patel W, Bradley WD, Block JE. Midterm outcomes of a prospective multicenter randomized controlled trial comparing the clinical efficacy of interspinous spacers as a treatment for moderate lumbar spinal stenosis. Spine J. 2013;13(9):S136-7.

15. Haley T, Miller LE, Block JE. Mid-term outcomes of a prospective randomized controlled trial of interspinous spacer treatment lumbar spinal stenosis. PM\&R. 2013;5(9):S287.

16. Blumenthal SL, Bradley DW Ohnmeiss DD. A prospective, randomized, multicenter study comparing two interspinous devices for the treatment of lumbar stenosis. Spine J. 2015;:S11

17. Gazzeri R, Galarza M, Alfieri A. Controversies about interspinous process devices in the treatment of degenerative lumbar spine diseases. past, present, and future. Biomed Res Int. 2014;2014:975052.

18. Kaulhausen T, Siewe J, Eysel P, Knifka K, Notermans HP, Koebke J, et al. The role of the inter-/supraspinous ligament complex in stand-alone interspinous process devices: a biomechanical and anatomic study. J Neurol Surg A Cent Eur Neurosurg. 2012;73(2):65-72.

19. Park SW, Lim TJ, Park J. A biomechanical stydy of the instrumented and adjacent lumbar levels after In-Space interspinous spacer insertion: laboratory investigation. J Neurosurg Spine. 2010;12(5):560-9.

20. Knowles FL. The Knowles vertebral support operation. Journal J lowa State Med Soc. 1958;48(10):551-4.

21. Sénégas J, Vital JM, Pointillart V, Mangione P. Clinical evaluation of a lumbar interspinous dynamic stabilization device (the Wallis system) with a 13-year mean follow-up. Neurosurg Rev. 2009;32(3):335-41.

22. Strömqvist BH, Berg S, Gerdhem P, Johnsson R, Möller A, Sahlstrand T, et al. X-STOP versus decompressive surgery for lumbar neurogenic intermittent claudication, randomized controlled trial with 2-year follow-up. Spine (Phila Pa 1976). 2013;38(17):1436-42.

Zucherman JF, Hsu KY, Hartjen CA, Mehalic TF, Implicito DA, Martin MJ, et al. A multicenter, prospective, randomized trial evaluating the X STOP terspinous process decompression system for the treatment of neurogenic intermittent claudication: two-year follow-up results. Spine (Phila Pa 1976). 2005:30(12):1351-8.

24. Lauryssen C, Jackson RJ, Baron JM, Tallarico RA, Lavelle WF, Deutsch H, et al. Stand-alone interspinous spacer versus decompressive laminectomy for treatment of lumbar spinal stenosis. Exp Rev Med Dev. 2015;12(6):763-9.

25. Parker SL, Anderson LH, Nelson T, Patel W, et al. Cost-effectiveness of three treatment strategies for lumbar spinal stenosis: conservative care, laminectomy, and the Superion interspinous spacer. Int J Spine Surg. 2015;9:28.

26. Burnett MG, Stein SC, Bartels RH. Cost-effectiveness of current treatment strategies for lumbar spinal stenosis: nonsurgical care, laminectomy, and XSTOP. J Neurosurg Spine. 2010;13(1):39-46.

27. Maida G, Marcati E, Sarubbo S. Heterotopic ossification in vertebral interlaminar, interspinous instrumentation: report of a case. Case Rep Surg. 2012;2012:970642.

28. Moojen WA, Arts MP, Jacobs WC, van Zwet EW, van den Akker-van Marle $M E$, Koes BW, et al. IPD without bony decompression versus conventional surgical decompression for lumbar spinal stenosis: 2-year results of a double-blind randomized controlled trial. Eur Spine J. 2015;24(10):2295-305.

29. Moojen WA, Arts MP, Jacobs WC, van Zwet EW, van den Akker-van Marle ME, Koes BW, et al. Interspinous process device versus standard conventional surgical decompression for lumbar spinal stenosis: randomized controlled trial. BMJ. 2013:347:f6415.

30. Wu AM, Zhou Y, Li QL, Wu XL, Jin YL, Luo P, et al. Interspinous spacer versus traditional decompressive surgery for lumbar spinal stenosis: a systematic review and meta-analysis. PLoS One. 2014;9(5):e97142.

31. Hong P, Liu Y, Li H. Comparison of the efficacy and safety between interspinous process distraction device and open decompression surgery in treating lumbar spinal stenosis: a meta analysis. J Investig Surg. 2015;28(1):40-9. 